\title{
Bridging the Pressure Gap in Electron Beam Induced Current Microscopy: Imaging Charge Transport in Metal Oxide Nanowires under Atmospheric Pressures
}

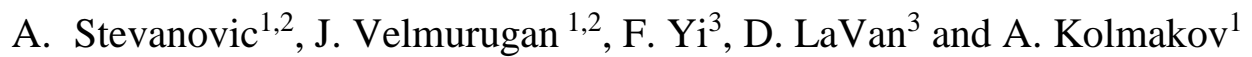 \\ 1. Center for Nanoscale Science and Technology, NIST, Gaithersburg, MD 20899 \\ 2. Maryland Nanocenter, University of Maryland, College Park, MD 20742 \\ 3. Material Measurement Laboratory, NIST, Gaithersburg, MD 20899
}

We investigate the charge transport in metal oxide semiconductor nanowires which are capable of converting solar light into electrical energy. The charge transport in semiconductors is influenced by electron donor or acceptor molecules adsorbed on a nanowire surface and by concentration of local defects affecting the work function and resulting in bending of the conduction and valence bands [1,2]. It is important to understand how this band bending in solid materials can be manipulated and optimized for the purposes of improving both photovoltaic (PV) cells and photocatalysts. In PV cells, band bending influences the charge transport along nanowires while in photocatalysis, the band bending governs the rates of photo-oxidation or photo-reduction reactions when electrons or holes are promoted to a surface. Therefore, the main objective is to characterize the dependence of charge transport through the nanowire and the chemical state of its surface.

We are developing a novel ambient pressure scanning electron microscopy (SEM) technique to study the interplay between surface reactivity and electronic properties of metal oxide nanowires such as $\mathrm{SnO}_{2}$, $\mathrm{TiO}_{2}$ and $\mathrm{ZnO}$. The main objective is to image electron beam induced current (EBIC) along a nanowire in the presence of oxidizing or reducing gas at atmospheric pressure. To bridge the "pressure gap" between high vacuum required for SEM operation and atmospheric pressure environment that houses the nanowire, we designed a measurement cell that is functional over a wide pressure range of $10^{-5} \mathrm{~Pa}$ to $10^{5} \mathrm{~Pa}$. An electron transparent $\mathrm{SiN}_{\mathrm{x}}$ membrane separates the two extremely different ambiences, atmospheric pressure region and high vacuum, allowing for both SEM and EBIC imaging as shown in Figure 1A. Figure 1B shows the SEM image of a single $50 \mu \mathrm{m}$ long and $0.22 \mu \mathrm{m}(220 \mathrm{~nm})$ thick $\mathrm{SnO}_{2}$ nanowire stretched between two platinum electrodes.

Here, we present the first imaging of the charge transport along $\mathrm{SnO}_{2}$ nanowire excited by $10 \mathrm{keV}$ electron beam under both vacuum and atmospheric pressure environments. We found that EBIC measurements are sensitive to adsorbates on $\mathrm{SnO}_{2}$ surface and electrode contacts that induce changes in the electronic structure of the semiconductor affecting its conductivity. Figure 2 shows the change in contrast in EBIC images under (A) vacuum and (B) air at $10^{5} \mathrm{~Pa}$. The change in EBIC contrast when the nanowire is exposed to air shows that electron transport is significantly reduced (gray scale is less bright compare to the vacuum measurement) likely due to the adsorption of oxygen on $\mathrm{SnO}_{2}$ surface that acts like an electron acceptor molecule. In addition, the I-V measurements reveal that the conductivity of the nanowire under vacuum is much higher (about three orders of magnitude) than in air. 
A.

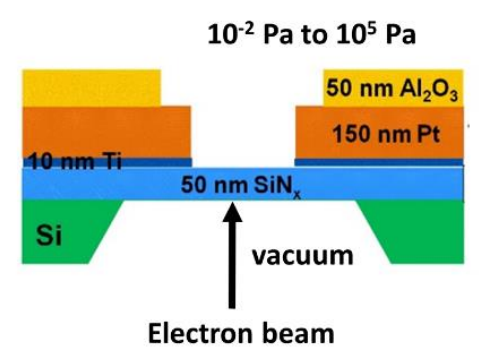

B.

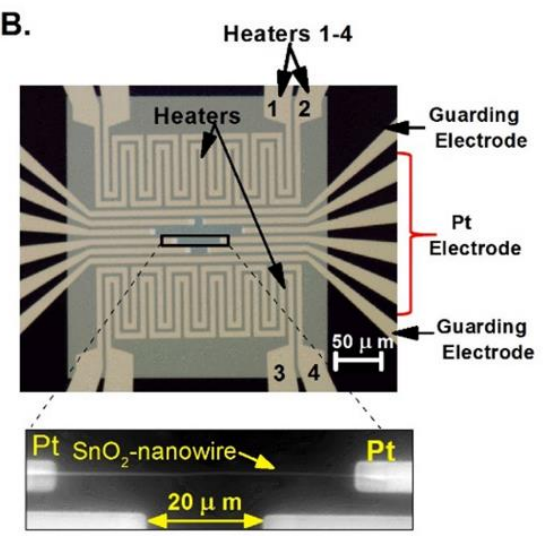

Figure 1. A. Fabrication of a micro cell - side view. A $50 \mathrm{~nm}$ thick $\mathrm{SiN}_{\mathrm{x}}$ electron transparent membrane (blue) which separates atmospheric from high vacuum conditions is deposited on the Si-chip. B. Electrodes layout. The micro sensor consists of Pt electrodes, Pt heaters and guarding electrodes [3]. The Pt electrodes can accommodate different nanowire lengths $(10 \mu \mathrm{m} ; 20 \mu \mathrm{m} ; 50 \mu \mathrm{m}$ and $100 \mu \mathrm{m})$ and the Pt heaters allow for uniform heating and cooling. The two guarding electrodes serve to block the electromagnetic field interference of the working heaters on electron beam induced current through a nanowire.
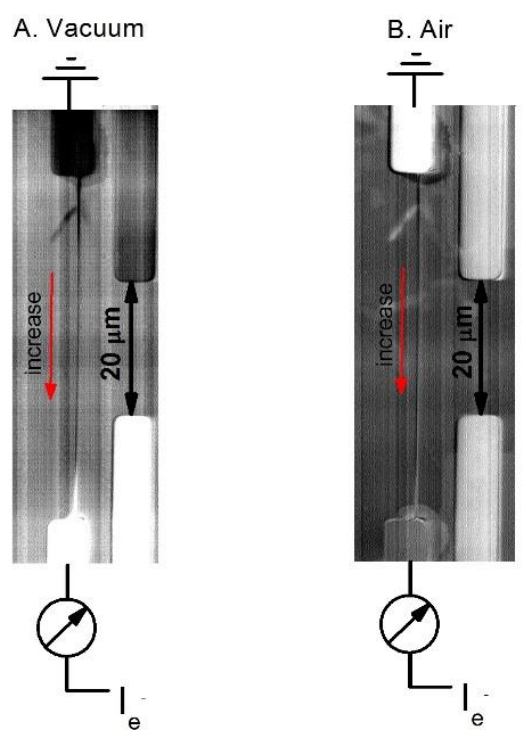

Figure 2. EBIC images of the charge transport through the $\mathrm{SnO}_{2}$ nanowire under A. vacuum and $\mathrm{B}$. air at $10^{5} \mathrm{~Pa}$. Both images show increase in EBIC contrast as electron beam approaches the non-grounded electrode and electronically excited electrons in $\mathrm{SnO}_{2}$ are more collected there. However, the EBIC image recorded in air shows less contrast than the image recoded under vacuum. This decrease in charge transport in air can be attributed to the adsorption of $\mathrm{O}_{2}$ that takes away excited electrons from the nanowire.

\section{References:}

[1] A Stevanovic et al, J. Am. Chem. Soc. 134 (2011), p. 324-332

[2] A Stevanovic et al, J. Phys. Chem. C 117 (2013), p. 24189-24195

[3] F Yi et al, J. Microelectromech. S. 24 (2015), p. 1185-1192 